\title{
Molecular characterization of the coagulase-negative staphylococcal surface flora of premature neonates
}

\author{
Hanna Bialkowska-Hobrzanska, ${ }^{1,3 *}$ Diane Jaskot ${ }^{3}$ and Ole Hammerberg ${ }^{1,2,3}$ \\ ${ }^{1}$ Departments of Microbiology and Immunology, and ${ }^{2}$ Pediatrics, University of Western Ontario, \\ ${ }^{3}$ Department of Microbiology and Infectious Diseases, St Joseph's Health Centre, 268 Grosvenor Street, London, \\ Ontario, Canada N6A $4 \mathrm{~V} 2$
}

(Received 4 May 1993; revised 23 August 1993; accepted 9 September 1993)

\begin{abstract}
A single point study was conducted to determine which surface sites best represent the density and composition of the coagulase-negative staphylococcal (CNS) colonizing flora in premature neonates. Five different surface sites of six randomly selected neonates hospitalized in a neonatal intensive care unit (NICU) for a month were examined. The individual strains and their clonal organization within CNS species were identified using restriction endonuclease fingerprinting of whole chromosomal DNA and ribosomal RNA genes. Cultures of the scalp, umbilicus, foot, nose and rectum were collected and quantitatively processed. Ten colonies were typed per surface culture. The most dense CNS colonization was noted on the umbilicus (mean $1.2 \times 10^{4} \mathrm{c.f} . \mathrm{u} . \mathrm{cm}^{-2}$ ), foot (mean $1.6 \times 10^{3}$ c.f.u. $\mathrm{cm}^{-2}$ ) and nose (mean $1.7 \times 10^{3}$ c.f.u. $\mathrm{cm}^{-2}$ ) of NICU neonates. Scalp and rectum were scarcely colonized. Of all the CNS surface isolates, $S$. epidermidis accounted for $77.7 \%(219 / 282)$ and $S$. haemolyticus, $S$. warneri and $S$. capitis accounted for $20.6 \%(58 / 282), 1.4 \%(4 / 282)$ and $0.4 \%(1 / 282)$, respectively. Colonization of each surface site comprised a maximum of five different strains representing four CNS species. Overall, five clones of $S$. epidermidis, two of $S$. haemolyticus, one of $S$. warneri and one of $S$. capitis were noted among the 282 isolates. The most predominant were two clones of $S$. epidermidis and one of $S$. haemolyticus; they accounted for $94 \%(265 / 282)$. Cultures from the foot and scalp represented the most heterogeneous CNS colonization of the five sites examined. Based on our findings of the existence of multiple strains of CNS at individual surface sites of NICU patients, we concluded that a minimum of five isolates be examined per surface culture to provide a comprehensive overview of the CNS colonizing flora.
\end{abstract}

\section{Introduction}

Coagulase-negative staphylococci (CNS) have become the most common cause of nosocomial bacteraemia in neonatal intensive care units (NICU) (Hall, 1991). They now account for approximately $90 \%$ of documented bacteraemias in the NICU at St. Joseph's Health Centre in London, Ontario. CNS constitute a major component of normal human skin and mucosal surface flora. These bacteria colonize all individuals, including neonates in NICU, within a few days of birth (D'Angio et al., 1989; Deighton et al., 1992; Hall et al., 1990; Valvano et al., 1988). Epidemiological investigations of CNS colon-

*Author for correspondence. Tel. +1 5196466000 ext. 5519; fax +15196466102 .

Abbreviations: CNS, coagulase-negative staphylococci; NICU, neonatal intensive care unit(s). ization and infection in NICU patients have been hampered by two factors; (i) the lack of specific methods for the identification of individual strains and their clonal relationships within a species, and (ii) limited information about the nature of CNS colonization at specialized surface sites of hospitalized newborns. Studies of natural populations of several important human pathogens have suggested a clonal composition of populations, comprising a relatively low total number of clones, with only a small fraction of clones associated with the disease (Selander et al., 1987). Clonal population structure is indicated by the persistence of identical or highly related strains among clinical isolates of a particular pathogen.

We have developed and evaluated molecular typing systems for CNS (Bialkowska-Hobrzanska et al., $1990 b, c)$. We have recently reported that analysis of chromosomal DNA restriction fragment length polymorphism is more reliable than plasmid profiling for 
differentiation of individual strains within a staphylococcal species (Bialkowska-Hobrzanska et al., 1990a), and have demonstrated the specificity of restriction endonuclease fingerprinting of ribosomal DNA for CNS speciation (Bialkowska-Hobrzanska et al., 1990c), which has also been reported by other groups (De Buyser $e t$ al., 1992). Earlier studies, using conventional methods for characterization of CNS flora in hospitalized adults, have shown the existence of a considerable number of distinct phage types of $S$. epidermidis on a single surface site (Heczko et al., 1978). The study described here employed molecular typing to characterize the CNS skin and mucosal surface flora of six NICU patients. We were particularly interested in determining the density of colonization and the number of distinct strains and species constituting CNS colonization patterns on various neonatal surface sites.

\section{Methods}

Patient selection. Patients were randomly selected and consisted of six neonates (birth weight $<1500 \mathrm{~g}$ ) admitted to the NICU of the St. Joseph's Health Centre between July and November 1990. This unit is a 42-bed facility and serves as a referral centre for low birth-weight newborns from Western Ontario in which approximately 20000 live births occur annually. Each neonate was sampled once between their 25 th and 30th day in the unit, which is when most predisposed neonates become bacteraemic with CNS (Freeman et al., 1990).

Source, identification and quantification of isolates. Five surface sites, consisting of the anterior nares, rectum, umbilicus, scalp and foot were sampled. Sterile cotton-tipped swabs (Kulsare; NCS Diagnostics) were premoistened with sterile $0.08 \mathrm{M}$-phosphate buffer, $\mathrm{pH} 7 \cdot 2$, and rubbed over approximately $1 \mathrm{~cm}^{2}$ area of surface for $5 \mathrm{sec}$. After sampling, the swabs were placed in sterile tubes containing $2 \mathrm{ml}$ Luria broth and transported into the laboratory. Further processing included vigorous vortexing $(\sim 10 \mathrm{~s}$ at high speed). Serial dilutions of each bacterial suspension were plated on $7 \%(\mathrm{v} / \mathrm{v})$ sheep-blood agar plates (Columbia agar, Oxoid). After incubation at $37^{\circ} \mathrm{C}$ for $24 \mathrm{~h}$ and then at room temperature for $48 \mathrm{~h}$, colonial morphology and the number of c.f.u. per site were determined. Identification of isolates was based on standard tests, colonial morphotypes, Gram stain, haemolytic pattern and coagulase test (Kloos et al., 1992). Gram-positive cocci that did not coagulate Bacto-Coagulase Plasma (Difco) after $2-4 \mathrm{~h}$ incubation at $37^{\circ} \mathrm{C}$ were referred to as CNS. All representative colonies from each morphotype, or up to ten random colonies per site if all appeared morphologically identical, were selected for examination by molecular typing. Isolates that were not identified by the automated Vitek System or identified with a confidence of at least $99 \%$ were identified to the species level by ribotyping (Bialkowska-Hobrzanska et al., 1990c). The following American Type Culture Collection type strains were used for comparison: S. epidermidis, ATCC 14990; S. haemolyticus, ATCC 29970; S. hominis, ATCC 27844; S. warneri, ATCC 27836; and S. cohnii, ATCC 29974.

Molecular typing. Restriction endonuclease fingerprinting of whole chromosomal DNA (chromosome typing) using ClaI was performed as previously described (Bialkowska-Hobrzanska et al., 1990a,b). Chromosomal DNA purified from representative isolates by caesium chloride-ethidium bromide gradient centrifugation was analysed for confirmation purposes. Restriction endonuclease fingerprinting of ribosomal RNA genes (ribotyping) consisted of DNA restriction with ClaI, separation of the fragments on agarose gels, transferring the fragments to a membrane, and hybridizing them with ${ }^{32} \mathrm{P}$-labelled $16+23 \mathrm{~S}$ rRNA from Escherichia coli as reported (BialkowskaHobrzanska et al., 1990 c). DNA patterns were scanned with a laser densitometer (Ultroscan XL model 2222, Pharmacia-LKB) and their genetic similarities were assessed using a computer program as described in a previous publication (Bialkowska-Hobrzanska et al., $1990 \mathrm{c}$ ). The criteria for the identity of isolates were based on the relatedness of chromosomal DNA banding patterns. Isolates with more than $95 \%$ DNA banding similarity were considered identical strains. Those with $85-95 \%$ similar bands were considered related strains. Both identical and related strains (with more than $85 \%$ similarity) were ascribed to the same clone. The isolates with less than $85 \%$ and more than $60 \%$ similarity were considered distinct strains. Plasmid profile analysis was conducted as previously described (Bialkowska-Hobrzanska et al., 1990a).

Antimicrobial susceptibility testing. Isolates representing distinct strains with different plasmid profiles were examined for their susceptibilities to the following antibiotics by automated Vitek testing: ampicillin, cephalothin, ciprofloxacin, clindamycin, erythromycin, oxacillin, penicillin, tetracycline, trimethoprim/sulphonamide, vancomycin.

\section{Results}

The density of CNS colonization was determined at three skin sites (the umbilicus, scalp and foot) and two mucosal sites (nose and rectum) of neonates hospitalized for a month in the NICU. The scalp, umbilicus and the foot were chosen as sampling sites to define the CNS flora at surface regions of potential entry of foreign bodies. The mucosal sites, known to support heavy bacterial colonization, were also examined to compare the composition of the CNS flora. Six neonates were included in this study. Cultures from the umbilicus, foot and nose represented the most dense CNS colonization (Table 1). The scalp and rectum were sparsely colonized.

To determine the composition of the CNS colonizing flora, 282 isolates from five surface sites of the six neonates (10 isolates per surface culture of a single site) were examined by chromosome typing and ribotyping.

Table 1. Density of CNS colonization on various skin and mucosal sites of premature newborns

\begin{tabular}{lcc}
\hline \hline & \multicolumn{2}{c}{$\begin{array}{c}10^{-2} \times \text { No. of CNS } \\
\left.\text { (c.f.u. } \text { cm }^{-2}\right)\end{array}$} \\
\cline { 2 - 3 } Site & Mean* & Range \\
\hline Umbilicus & 123 & $4-7200$ \\
Nose & 17 & $1-140$ \\
Foot & 16 & $4-2100$ \\
Scalp & $0 \cdot 3$ & $0 \cdot 04-2$ \\
Rectum & 0.3 & $0.01-5$ \\
\hline \hline
\end{tabular}

${ }^{*}$ Geometric means were used to describe total CNS counts expressed in c.f.u. 
Table 2. Colonization patterns of CNS on various skin and mucosal sites of premature newborns

A numerical system has been introduced to describe individual strains of CNS. A specific ribosomal DNA banding pattern (ribotype) combined with chromosomal DNA pattern (chromosome type) is referred to as a molecular type. Three numerals separated by periods are assigned to denote molecular type. The first numeral (1-15) represents species-specific ribotype. For example, the first numerals 5, 7, 14 and 2 represent $S$. epidermidis, $S$. haemolyticus, $S$. warneri and $S$. capitis, respectively. The second and third numerals denote strain-specific chromosome types. The same second numeral indicates identical or related strains, with more than $85 \%$ similarity, representing the same clone. Different second numerals indicate distinct strains, with $60-85 \%$ similarity. The third numeral refers to strain variants within a given clone.

\begin{tabular}{cccccc}
\hline \hline & \multicolumn{5}{c}{ Molecular type (no. of isolates) per site } \\
\cline { 2 - 6 } Newborn & Scalp & Umbilicus & Foot & Nose & Rectum \\
\hline \multirow{2}{*}{1} & $5.2 .1(9)$ & $5.2 .1(1)$ & $5.3 .2(2)$ & $5.2 .1(5)$ & \\
& $5.3 .1(1)$ & $5.3 .1(4)$ & $5.5 .1(2)$ & $5.3 .1(2)$ & \\
& & $7.1 .1(5)$ & $7.1 .1(1)$ & $7.2 .1(3)$ & \\
& & & $14.1 .1(4)$ & & \\
\multirow{2}{*}{2} & & & $2.1 .1(1)$ & & \\
& $5.2 .1(4)$ & $5.2 .1(4)$ & $5.2 .1(3)$ & $5.2 .1(1)$ & $5.3 .1(2)$ \\
& $5.3 .1(5)$ & $5.3 .1(2)$ & $5.3 .1(3)$ & $5.3 .1(7)$ & $7.1 .1(8)$ \\
3 & $5.1 .1(1)$ & $7.1 .1(4)$ & $7.1 .1(4)$ & $5.1 .1(2)$ & \\
& $5.2 .1(3)$ & $5.2 .1(6)$ & $5.3 .1(5)$ & $5.2 .1(3)$ & $5.2 .1(1)$ \\
& $5.3 .1(3)$ & $5.3 .1(1)$ & $7.1 .1(4)$ & $5.3 .1(3)$ & $5.3 .1(8)$ \\
& $5.1 .1(3)$ & $7.1 .1(3)$ & $7.1 .2(1)$ & $7.1 .1(4)$ & $7.1 .1(1)$ \\
4 & $7.1 .2(1)$ & & & & \\
& $5.2 .1(8)$ & $5.2 .1(7)$ & $5.2 .1(7)$ & $5.2 .1(7)$ & $5.2 .1(1)$ \\
& $5.3 .1(1)$ & $5.3 .1(2)$ & $5.3 .1(1)$ & $5.3 .1(2)$ & $5.3 .1(2)$ \\
& $5.6 .1(1)$ & $7.1 .1(1)$ & $7.1 .1(2)$ & $7.1 .1(1)$ & $7.1 .1(7)$ \\
5 & $5.3 .1(2)$ & $5.3 .1(10)$ & $5.3 .1(10)$ & $5.3 .1(10)$ & $5.3 .1(10)$ \\
6 & $5.2 .1(8)$ & $5.2 .1(6)$ & $5.2 .1(3)$ & $5.3 .1(10)$ & $5.3 .1(9)$ \\
& $5.2 .2(2)$ & $5.3 .1(2)$ & $5.3 .1(2)$ & & $7.1 .1(1)$ \\
& & $7.1 .1(2)$ & $7.1 .1(5)$ & & \\
\hline \hline
\end{tabular}

Colonization patterns of skin and mucosal surface sites of the six neonates are summarized in Table 2. S. epidermidis accounted for $77.7 \%(219 / 282)$ of the CNS flora. S. haemolyticus, $S$. warneri and $S$. capitis accounted for $20.6 \%(58 / 282), 1.4 \%(4 / 282)$ and $0.4 \%(1 / 282)$, respectively. Overall, five distinct clones of $S$. epidermidis, two of $S$. haemolyticus, one of $S$. warneri and one of $S$. capitis were detected (Fig. 1; Table 2).

Ribotyping distinguished four distinctive rDNA patterns (ribotypes) among 219 S. epidermidis isolates (Fig. $1 c$, lanes 1-5). Single rDNA patterns were identified among $58 \mathrm{~S}$. haemolyticus isolates, four $S$. warneri isolates and one $S$. capitis isolate (Fig. $1 c$, lanes 6-9). The ribotype 2 was the most prevalent among $S$. epidermidis isolates comprising the two clones 5.2 and 5.3. The relatedness of chromosomal DNA banding patterns between clones constituting ribotype 2 was found to be greater than among clones representing distinct ribo- types. Thus, ribotyping, although less discriminatory than chromosome typing for identification of individual strains (Bialkowska-Hobrzanska et al., 1990c), was useful for 'clustering' of clones.

When comparing colonial morphotypes among distinct strains of CNS we found that four strains of $S$. epidermidis (designated 5.2.1, 5.3.1, 5.5.1 and 5.6.1) yielded non-haemolytic, same size, greyish-white colonies with similar density and consistency. The same morphotype, with the exception of a slightly larger colony size, was also typical for $S$. warneri strain 14.1.1. This indicates that selection based only on distinct colonial morphotypes could underestimate the total number of CNS strains among surface cultures.

Cultures from the foot and scalp yielded the most heterogeneous CNS flora of the five sites examined. As many as five distinct strains, which represented species of S. epidermidis, S. haemolyticus, S. warneri and S. capitis, were recovered from the foot. $S$. epidermidis was, quantitatively, the predominant species found on all surface sites examined. Two clones of $S$. epidermidis $(5.2$ and 5.3) accounted for $74.5 \%$ of the total of $282 \mathrm{CNS}$ isolates collected from all sites of the six neonates. One $S$. epidermidis clone, 5.2, comprising two closely related strains 5.2 .1 and 5.2.2, constituted $25.2 \%$ of the total CNS flora at the scalp, umbilicus and foot. The two clones of $S$. epidermidis (5.2 and 5.3) and one of $S$. haemolyticus (7.1) were simultaneously isolated from $56.7 \%(17 / 30)$ of the surface sites of six neonates. Quantitatively, S. epidermidis clones other than 5.2 and 5.3 were present in low numbers. For instance, $S$. epidermidis 5.6 and $S$. epidermidis 5.1 accounted for $1.9 \%(1 / 52)$ and $7.7 \%(4 / 52)$ only, respectively, of all CNS isolates recovered from the scalp of six neonates.

There was a good correlation between patterns of chromosomal DNA and plasmid profiles for S. haemolyticus strain 7.1.1 and S. epidermidis strain 5.2.1. Of the S. epidermidis isolates with chromosome type $5.2 .1,98 \%$ carried plasmids of molecular size $2.3 \mathrm{~kb}, 4.4 \mathrm{~kb}, 8.7 \mathrm{~kb}$, $13.5 \mathrm{~kb}$ and $27 \mathrm{~kb}$. Three plasmids of the same molecular size $(2.3 \mathrm{~kb}, 4.4 \mathrm{~kb}, 8.7 \mathrm{~kb})$ were harboured by all $S$. haemolyticus isolates with chromosome type 7.1.1. In contrast, S. epidermidis strain 5.3.1 displayed five distinct plasmid profiles. S. epidermidis 5.3.1, carrying plasmids of different molecular size, were isolated most frequently from different NICU patients or from different surface sites of the same neonate; occasionally such strains were isolated from the same neonatal site.

All CNS isolates examined in this study, except for a single $S$. capitis isolate, were resistant to multiple antibiotics, but all strains remained sensitive to vancomycin (Table 3). Most S. haemolyticus isolates were resistant to eight of the antibiotics tested and the $S$. epidermidis isolates were resistant to at least six. 
(a)

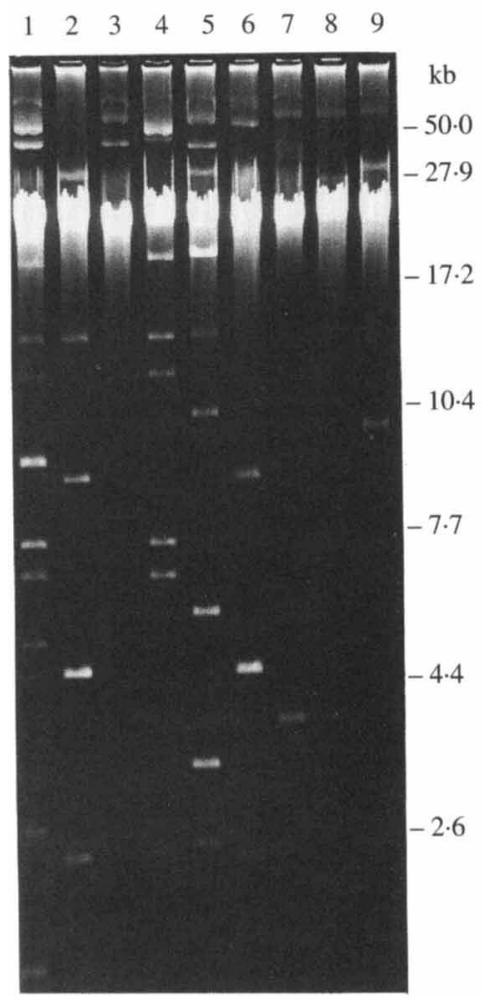

(b)

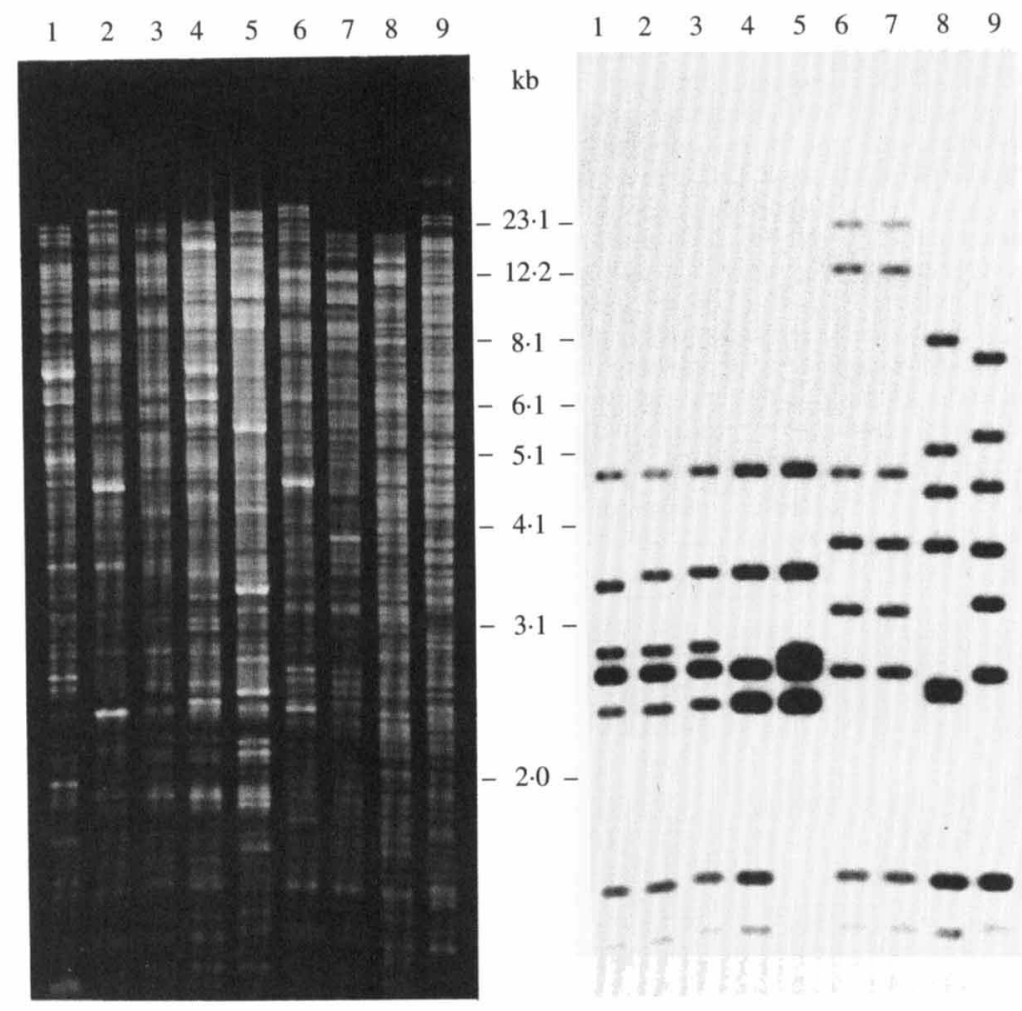

Fig. 1. DNA patterns of representative strains of CNS. (a) Plasmid profile analysis; (b) chromosome typing; (c) ribotyping. Lanes: 1, S. epidermidis $5.1 .1 ; 2$, S. epidermidis $5.2 .1 ; 3$, S. epidermidis $5.3 .1 ; 4$, S. epidermidis $5.5 .1 ; 5$, S. epidermidis $5.6 .1 ; 6$, S. haemolyticus $7.1 .1 ; 7$, S. haemolyticus $7.2 .1 ; 8$, S. warneri $14.1 .1 ; 9$, S. capitis 2.1.1.

Table 3. Antibiograms of CNS surface culture isolates from neonates residing in NICU for a month

The definition of resistance is based on the MICs for: Ap, ampicillin $\left(\geqslant 32 \mu \mathrm{g} \mathrm{m}^{-1}\right) ;$ Ce, cephalothin $\left(\geqslant 32 \mu \mathrm{g} \mathrm{ml}^{-1}\right)$; $\mathrm{Ci}$, ciprofloxacin $\left(\geqslant 2 \mu \mathrm{g} \mathrm{ml}^{-1}\right)$; Cli, clindamycin $\left(\geqslant 8 \mu \mathrm{g} \mathrm{ml}^{-1}\right)$; E, erythromycin $\left(\geqslant 8 \mu \mathrm{g} \mathrm{ml}^{-1}\right) ; \mathrm{Ox}$, oxacillin $\left(\geqslant 4 \mu \mathrm{g} \mathrm{ml}^{-1}\right) ; \mathrm{P}$, penicillin $\left(\geqslant 16 \mu \mathrm{g} \mathrm{ml}^{-1}\right) ; \mathrm{T}$, tetracycline $\left(\geqslant 16 \mu \mathrm{g} \mathrm{m}^{-1}\right) ; \mathrm{T} / \mathrm{S}$, trimethoprim/sulphonamide $\left(\geqslant 4 / 76 \mu \mathrm{g} \mathrm{ml}^{-1}\right) ; \mathrm{V}$, vancomycin $\left(\geqslant 6 \mu \mathrm{g} \mathrm{ml}^{-1}\right)$.

\begin{tabular}{lccccccccccc}
\hline \hline & \multicolumn{10}{c}{ Percentage of isolates resistant to: } \\
\cline { 2 - 10 } $\begin{array}{l}\text { Organism } \\
\text { (no. of isolates) }\end{array}$ & $\mathrm{Ap}$ & $\mathrm{Ce}$ & $\mathrm{Ci}$ & $\mathrm{Cli}$ & $\mathrm{E}$ & $\mathrm{Ox}$ & $\mathrm{P}$ & $\mathrm{T}$ & $\mathrm{T} / \mathrm{S}$ & $\mathrm{V}$ \\
\hline $\begin{array}{l}\text { S. epidermidis } \\
(49)\end{array}$ & 100 & 100 & 6 & 92 & 100 & 100 & 100 & 0 & 51 & 0 \\
$\begin{array}{l}S . \text { haemolyticus } \\
(26)\end{array}$ & 100 & 100 & 0 & 85 & 92 & 100 & 100 & 77 & 85 & 0 \\
$\begin{array}{c}\text { S. warneri } \\
(2)\end{array}$ & 50 & 50 & 0 & 50 & 100 & 50 & 100 & 0 & 0 & 0 \\
$\begin{array}{c}S . \text { capitis } \\
(1)\end{array}$ & 0 & 0 & 0 & 0 & 0 & 0 & 0 & 0 & 0 & 0 \\
\hline \hline
\end{tabular}

\section{Discussion}

The present study represents the first comprehensive survey of CNS colonizing flora in NICU patients using molecular typing. There were three major findings in this study. First, molecular examination of a large number of isolates from individual surface sites revealed the existence of multiple chromosomal DNA patterns among colonies of similar morphology. Second, this approach demonstrated that colonization patterns of individual neonatal skin and mucosal sites comprised up to five distinct strains representing four CNS species, $S$. epidermidis, S. haemolyticus, S. warneri and S. capitis. Third, there were two clones of $S$. epidermidis and one of $S$. haemolyticus which predominated quantitatively on five infants, suggesting nosocomial acquisition.

The highest density of CNS colonization (mean $1 \cdot 2 \times 10^{4}$ c.f.u. $\mathrm{cm}^{-2}$ ) was observed on the umbilicus. A similar density of CNS colonization was previously noted in the oropharynx of 14 NICU patients undergoing antibiotic therapy (Sprunt et al., 1978). A significantly lower titre of viable CNS (mean of 30 c.f.u. $\mathrm{cm}^{-2}$ ) recovered from the posterior surface of the scalp of the neonates may reflect nursing practices of holding neonates with hands washed in antiseptics. Leyden (1982) found denser CNS colonization $\left(1.8 \times 10^{5}\right.$ c.f.u. $\mathrm{cm}^{-2}$ ) on the scalp of 6-week-old healthy full-term infants. 
This investigation demonstrated the existence of a clonal structure of CNS flora. Analysis of restriction fragment length polymorphism patterns of whole chromosomal DNA resulted in the identification of individual strains and clonal relationships among CNS strains. A clone was arbitrarily defined as a set of strains showing identical or related chromosomal DNA patterns with more than $85 \%$ similarity, thus suggesting their common ancestry. Ribotyping confirmed the clonal organization of CNS species determined by chromosome typing; clonal strain variants always had the same restriction fragment length polymorphism patterns of rDNA. This method permitted 'clustering' of related clones within the species; clones classified into the same ribotype displayed greater similarity between their chromosomal DNA patterns than clones representing distinct ribotypes. Clonal organization of $S$. capitis isolated from healthy adults has previously been documented on the basis of analysis of total protein patterns (Maggs \& Pennington, 1989).

The composition of the CNS flora at the umbilical, nasal and rectal sites of neonates was virtually identical and comprised two out of five clones of $S$. epidermidis and two of two $S$. haemolyticus. The additional three clones of $S$. epidermidis and the single clones of $S$. warneri and $S$. capitis were noted only on the scalp and feet. Whether the difference in colonization patterns of examined neonatal surface sites reflects specialization of the CNS flora or merely reflects greater exposure to the environment remains to be determined. Previous reports on the natural history of CNS colonization in full-term infants indicated a largely uniform distribution of CNS species and biotypes (Carr \& Kloos, 1977).

The demonstration of the same two clones of $S$. epidermidis and one of S. haemolyticus on surface sites of five out of the six neonates indicated a common nosocomial source. These clones have previously been observed in our NICU (Bialkowska-Hobrzanska et al., $1990 a$ ). The evidence documenting the endemic character of these prevalent CNS clones has been described elsewhere (Bialkowska-Hobrzanska et al., 1991). The hospitalization times of some of the neonates examined in this study overlapped, which probably contributed to the acquisition of the same CNS clones. Our recent work has demonstrated that the NICU staff carried endemic CNS clones in the anterior nares (data not shown). We have also sequentially sampled eight neonates after admission to the NICU during 1991. All infants become colonized with endemic CNS clones within the first week of admission (unpublished data). D'Angio et al. (1989) reported the predominance of a single biotype of $S$. epidermidis among the surface flora of the NICU patients. In view of this and several previous studies (Bialkowska-Hobrzanska et al., $1990 a$; Nystrom et al.,
1992; Renaud et al., 1988) it is possible that this biotype may, in fact, contain distinct strains.

A comparison of the relative percentage of the CNS species, determined by ribotyping, has confirmed the prevalence of $S$. epidermidis followed by $S$. haemolyticus. An earlier study by Goldmann et al. (1978) recognized that almost all infants admitted to NICU were colonized with $S$. epidermidis. Hall et al. (1990) also found $S$. epidermidis predominated among CNS nasopharyngeal cultures from premature neonates. The involvement of other CNS species, S. haemolyticus $(21 \%), S$. warneri $(1 \%)$ and S. capitis $(1 \%)$, established in their study (Hall et al., 1990), is in agreement with this study, despite different methods used for CNS speciation.

The processing of specimens employed in this study and the testing of a large number of colonies of similar morphology, ensured that a representative fraction of the colonizing flora of a specialized site on a neonate was analysed. Quantitative recovery of micro-organisms from the swab, and typing of 10 colonies per surface culture, permitted us to detect multiple CNS strains on individual surface sites. Occasionally, all 10 colonies had indistinguishable morphotypes on sheep-blood agar plates, yet they represented distinct strains. We found that as many as four distinct strains of $S$. epidermidis yielded similar colony morphotypes. Colonial variants of the same strain, S. epidermidis 5.2.1, which differed slightly in colonial morphology on Congo red blood agar plates and in their haemolytic and proteolytic phenotypes were noted in this study. They resembled recently described $S$. epidermidis colonial variants (Deighton et al., 1992). Previous epidemiological studies of CNS colonization and infections analysed only colonies representing distinct colonial morphotypes (Mehta et al., 1991 ; Nystrom et al., 1992).

Antibiotic multiresistance of $S$. epidermidis and $S$. haemolyticus from the NICU environment, as observed in this study, is consistent with previous observations. All strains of these species from our NICU were resistant to methicillin and gentamicin. According to a recent report (Hall et al., 1988), 86\% of $S$. epidermidis and $S$. haemolyticus isolates causing neonatal bacteraemia were resistant to methicillin. Similar data have been described by others (Noel \& Edelson, 1984; Davies et al., 1986). It is likely that routine treatment of most NICU patients with ampicillin and gentamicin selects for multiresistant clones.

A good correlation was noted between chromosomal restriction fragment length polymorphism and the plasmid content of the most abundant clones of $S$. epidermidis and of $S$. haemolyticus. Interestingly, these two clones, which persisted in our NICU over the past 6 years, carried three plasmids of identical molecular size $(2.3 \mathrm{~kb}, 4.4 \mathrm{~kb}, 8.7 \mathrm{~kb})$. Studies are in progress to 
determine whether these plasmids contribute to increased pathogenicity and persistence of certain CNS clones in the NICU patients.

This study employed a molecular typing system for classification of individual strains and clones and the establishment of clonal relationships within CNS species. It is hoped such an approach may be useful in population genetics studies, contributing to the understanding of the pathogenicity of CNS.

This study was supported by grants from the Richard and Jean Ivy Fund and the Hospital for Sick Children Foundation (grant no. XG81010). We thank Miguel Valvano for critical reading of the manuscript, Wei Zhong for contributing to molecular typing of isolates, the Bacteriology Laboratory for antimicrobial susceptibility testing, and Infection Control nurses for collecting swabs from NICU patients.

\section{References}

Bialkowska-Hobrzanska, H., Jaskot, D. \& Hammerberg, O. $(1990 \mathrm{a})$. Evaluation of restriction endonuclease fingerprinting of chromosomal DNA and plasmid profile analysis for characterization of multiresistant coagulase-negative staphylococci in bacteraemic neonates. Journal of Clinical Microbiology 28, 269-275.

Bialkowska-Hobrzanska, H., Jaskot, D. \& Hammerberg, O. $(1990 b)$. A method for DNA restriction endonuclease fingerprinting of coagulase-negative staphylococci. Journal of Microbiological Methods 12, 41-49.

Bialkowska-Hobrzanska, H., Harry, V., Jaskot, D. \& HammerBERG, O. $(1990 c)$. Typing of coagulase-negative staphylococci by Southern hybridization of chromosomal DNA fingerprints using an rRNA gene probe. European Journal of Clinical Microbiology \& Infectious Diseases 9, 588-594.

Bialkowska-Hobrzanska, H., Jaskot, D., Zhong, W., Harry, V. S. \& HAMmerberg, O. (1991). Molecular characterization of endemic coagulase-negative staphylococcal isolates from surface sites of hospitalized premature neonates. Recent Advances in Chemotherapy, ICC Proceedings, p. 1140.

CARR, D. L. \& KLoos, E. (1977). Temporal study of the staphylococci and micrococci of normal infant skin. Applied and Environmental Microbiology 34, 673-680.

D'Angio, C. T., McGowan, K. L., Baumgart, S., St. Geme, J. \& HARRIS, M. C. (1989). Surface colonization with coagulase-negative staphylococci in premature neonates. Journal of Pediatrics 114, 1029-1034.

Davis, A. J., Clevet, J. \& Jones, A. (1986). Sensitivity patterns of coagulase-negative staphylococci from neonates. Journal of Antimicrobial Chemotherapy 17, 155-160.

De Buyser, M.-L., Morvan, A., Aubert, S., Dilasser, F, \& El Solh, N. (1992). Evaluation of a ribosomal RNA gene probe for the identification of species and subspecies within the genus Staphylococcus. Journal of General Microbiology 138, 889-899.

Deighton, M., Pearson, S., Capstick, J., Spelman, D. \& Borland, R.
(1992). Phenotypic variation of Staphylococcus epidermidis isolated from a patient with native valve endocarditis. Journal of Clinical Microbiology 30, 2385-2390.

Freeman, J., Epstein, M. F., Smith, N. E., Platt, R., Sidebottom, D. G. \& GoldmanN, D. A. (1990). Extra hospital stay and antibiotic usage with nosocomial coagulase-negative staphylococcal bacteraemia in two neonatal intensive care unit populations. American Journal of Diseases of Children 144, 324-329.

Goldmann, D. A., Leclair, J. \& Macone, A. (1978). Bacterial colonization of neonates admitted to an intensive care environment. Journal of Pediatrics 93, 288-293.

HALL, S. L. (1991). Coagulase-negative staphylococcal infections in neonates. Pediatric Infectious Disease Journal 10, 57-67.

Hall, S. L., Hall, R. T., Barns, W. G. \& Riddell, S. (1988). Colonization with slime-positive coagulase-negative staphylococci as a risk factor for invasive coagulase-negative staphylococcal infections in neonates. Journal of Perinatology 8, 215-221.

Hall, S. L., Riddell, S. C., Barnes, W. G., Meng, L. \& Hall, R. T. (1990). Evaluation of coagulase-negative staphylococcal isolates from serial nasopharyngeal cultures of premature infants. Diagnostic Microbiology and Infectious Disease 13, 17-23.

Heczko, P. B., Klein, A., Kasprowicz, A. \& Pulverer, G. (1978). Use of a phage set for the ecological typing of coagulase-negative staphylococci. Zentralblatt für Bakteriologie Parasitenkunde, Infektionskrankheiten und Hygiene (Abt. I, Originale A) 241, 157-162.

KLoos, W. E., Schleifer, K.-H. \& GöTZ, F. (1992). The genus Staphylococcus. In The Prokaryotes, 2nd edn, vol. 2, pp. 1369-1420. Edited by A. Balows, H. G. Trüper, M. Dworkin, W. Harder \& K.-H. Schleifer. New York: Springer-Verlag.

LEYDEN, J. L. (1982). Neonatal skin structure and function-bacteriology of newborn skin. Dermatology Series 1, 167-181.

MagGs, A. F. \& Pennington, T. H. (1989). Temporal study of staphylococcal species on the skin of human subjects in isolation and clonal analysis of Staphylococcus capitis by sodium dodecyl sulfatepolyacrylamide gel electrophoresis. Journal of Clinical Microbiology 27, 2627-2632.

MeHTA, G., Singh, S. \& KumarI, S. (1991). Observations on coagulasenegative staphylococci in a neonatal unit in India. Journal of Hospital Infection 19, 273-281.

Noel, G. J. \& Edelson, P. J. (1984). Staphylococcus epidermidis bacteraemia in neonates: further observations and the occurrence of focal infection. Pediatrics 74, 832-839.

Nystrom, B., Ransjo, U., Ringertz, S., Faxelius, G., Tunell, R., Ohman, G., Wilton, J. \& Pfaller, M. A. (1992). Colonization with coagulase-negative staphylococci in two neonatal units. Journal of Hospital Infection 22, 287-298.

Renaud, F., Freney, J., Etienne, J., Bes, M., Brun, Y., Barsotti, O., ANDRE, S. \& FLEURETTE, J. (1988). Restriction endonuclease analysis of Staphylococcus epidermidis DNA may be a useful epidemiological marker. Journal of Clinical Microbiology 29, 1729-1734.

Selander, R. K., Musser, J. M., Caugant, D. A., Gilmour, M. N. \& WhitTam, T. S. (1987). Population genetics of pathogenic bacteria. Microbial Pathogenesis 3, 1-7.

Sprunt, K., LeIDy, G. \& Redman, W. (1978). Abnormal colonization of neonates in an intensive care unit: means of identifying neonates at risk of infection. Pediatric Research 12, 998-1002.

Valvano, M. A., Hartstein, A. I., Morthland, V. H., Dragoon, M. E., Potter, S. A., Reynolds, J. W. \& Crosa, J. H. (1988). Plasmid DNA analysis of Staphylococcus epidermidis isolated from blood and colonization cultures in very low birth weight neonates. Pediatric Infectious Disease Journal 7, 116-120. 